\title{
A nomogram for predicting the risk of lymph node metastasis in T1-2 non-small-cell lung cancer based on PET/CT and clinical characteristics
}

\author{
Xiayi $\mathbf{L v}^{1 \#}$, Zhigang $\mathrm{Wu}^{1 \#}$, Jinlin $\mathrm{Cao}^{1 \#}$, Yeji Hu${ }^{1}$, Kai Liu ${ }^{2}$, Xiaona Dai ${ }^{3}$, Xiaoshuai Yuan ${ }^{1}$, Yiqing Wang \\ Kui Zhao ${ }^{4}$, Wang $\mathrm{Lv}^{1}$, Jian $\mathrm{Hu}^{1}$ \\ ${ }^{1}$ Department of Thoracic Surgery, The First Affiliated Hospital, School of Medicine, Zhejiang University, Hangzhou, China; ${ }^{2}$ Department of \\ Thoracic Surgery, Sir Run Run Shaw Hospital, School of Medicine, Zhejiang University, Hangzhou, China; ${ }^{3}$ Department of Quality Management, \\ The Second Affiliated Hospital, School of Medicine, Zhejiang University, Hangzhou, China; ${ }^{4}$ Departments of Radiology, The First Affiliated \\ Hospital, School of Medicine, Zhejiang University, Hangzhou, China \\ Contributions: (I) Conception and design: J Cao, J Hu; (II) Administrative support: X Lv, J Hu; (III) Provision of study materials or patients: K Liu, K \\ Zhao; (IV) Collection and assembly of data: X Lv, Z Wu, Y Hu, K Liu; (V) Data analysis and interpretation: J Cao, X Dai, X Yuan, Y Wang, W Lv; (VI) \\ Manuscript writing: All authors; (VII) Final approval of manuscript: All authors. \\ \#These authors contributed equally to this work. \\ Correspondence to: Prof. Jian Hu. Department of Thoracic Surgery, The First Affiliated Hospital, School of Medicine, Zhejiang University, 79 \\ Qingchun Road, Hangzhou 310003, China. Email: dr_hujian@zju.edu.cn.
}

Background: Accurately predicting the risk level for a lymph node metastasis is critical in the treatment of non-small cell lung cancer (NSCLC). This study aimed to construct a novel nomogram to identify patients with a risk of lymph node metastasis in T1-2 NSCLC based on positron emission tomography/computed tomography (PET/CT) and clinical characteristics.

Methods: From January 2011 to November 2017, the records of 318 consecutive patients who had undergone PET/CT examination within 30 days before surgical resection for clinical T1-2 NSCLC were retrospectively reviewed. A nomogram to predict the risk of lymph node metastasis was constructed. The model was confirmed using bootstrap resampling, and an independent validation cohort contained 156 patients from June 2017 to February 2020 at another institution.

Results: Six factors [age, tumor location, histology, the lymph node maximum standardized uptake value (SUVmax), the tumor SUVmax and the carcinoembryonic antigen (CEA) value] were identified and entered into the nomogram. The nomogram developed based on the analysis showed robust discrimination, with an area under the receiver operating characteristic curve of 0.858 in the primary cohort and 0.749 in the validation cohort. The calibration curve for the probability of lymph node metastasis showed excellent concordance between the predicted and actual results. Decision curve analysis suggested that the nomogram was clinically useful.

Conclusions: We set up and validated a novel and effective nomogram that can predict the risk of lymph node metastasis for individual patients with T1-2 NSCLC. This model may help clinicians to make treatment recommendations for individuals.

Keywords: Non-small cell lung cancer (NSCLC); positron emission tomography/computed tomography (PET/ CT); model; nomogram; lymph node status

Submitted Jul 24, 2019. Accepted for publication Oct 24, 2020.

doi: $10.21037 /$ tlcr-20-1026

View this article at: http://dx.doi.org/10.21037/tlcr-20-1026 


\section{Introduction}

Nodal staging is of concern to clinicians who treat patients with non-small cell lung cancer (NSCLC), as it affects patients' prognosis and guides their therapeutic strategies $(1,2)$. Patients deemed to have clinical T1-2N0 NSCLC may be offered limited surgical resection or stereotactic body radiotherapy, particularly for patients with multiple comorbidities and poor lung function that limit optimal treatment planning (3-5). However, how to accurately predict the nodal status before pathological confirmation is a challenge to clinicians. Invasive procedures such as mediastinoscopy, endobronchial ultrasound transbronchial needle aspiration and trans-oesophageal needle aspiration may in some cases yield pathological diagnoses, but it is difficult to obtain biopsies that contain several groups of nodes. The risk of complications after biopsy is particularly high for patients with asthenia $(6,7)$.

Many scholars have attempted to predict nodal status in patients with NSCLC based on imaging characteristics before invasive procedures or selection of patients for invasive nodal staging (8-10). In many centers, $18 \mathrm{~F}$-fluorodeoxyglucose positron emission tomography/ computed tomography (PET/CT) imaging is the most common method of diagnosing and staging NSCLC. Alternative imaging criteria for malignancy have often been suggested to improve sensitivity and specificity; however, it has been difficult to find which factors are most important in predicting nodal metastasis $(6,7,11,12)$. It is thus of great clinical significance to find an effective and practical approach to predict nodal metastasis in patients with NSCLC.

Nomograms have been accepted as reliable tools for use with a variety of malignancies to enhance outcome prediction and quantify risk when making treatmentrelated decisions $(13,14)$. Two prediction models have been established to estimate the pretest probability of N2 lymph nodes metastasis in patients with NSCLC based on PET/ CT $(7,15)$, and only one model incorporated four factors have been built to predict the lymph node involvement in NSCLC based on PET/CT (16). However, the latter model lack of validation and the pathological results come from biopsies (16). In this study, we constructed and validated a nomogram that combines patients' characteristics with the findings of PET/CT examination to predict lymph node metastasis in patients who had undergone surgical resection for clinical T1-2 NSCLC. We present the following article in accordance with the TRIPOD reporting checklist (available at http://dx.doi.org/10.21037/tlcr-20-1026).

\section{Methods}

\section{Patients}

The primary cohort of this study comprised 318 consecutive patients who had undergone PET/CT before surgical resection for clinical T1-2 NSCLC at the First Affiliated Hospital of Zhejiang University between January 2011 and November 2017. The model was confirmed using an independent validation cohort contained 156 patients from June 2017 to February 2020 at Sir Run Run Shaw Hospital. The inclusion criteria were as follows: (I) patients had undergone 18F-fluorodeoxyglucose PET-CT in the 30 days before surgical resection; (II) the patients' blood glucose levels had been controlled at a normal level $(<6.8 \mathrm{~mL} / \mathrm{L})$; (III) patients had undergone no neoadjuvant chemotherapy or radiation before PET/CT examination [to ensure that the maximum standardized uptake value (SUVmax) was not affected by radiotherapy or chemotherapy]. Systematic lymph node dissection or sampling was carried out at stations \#2, \#3, \#4, \#7, \#8, \#9, \#10 and \#11 for rightsided tumors and at stations \#5, \#6, \#7, \#8, \#9, \#10 and \#11 for left-sided tumors. A central tumor was defined as existing within the proximal third of the hemithorax, and a peripheral tumor was defined as existing outside the proximal third of the hemithorax (13).

The study was performed following the principles outlined in the Declaration of Helsinki (as revised in 2013). The study was approved by the university's ethical committee and institutional review board. (No.2020759) and written informed consent was obtained from all patients.

\section{PET-CT examination}

PET/CT was conducted in all patients with an integrated PET/CT scanner (Biograph-16, Siemens, Germany) within the 30 days before surgery. Baseline scans of the chest were performed using a 5 -mm section thickness of the slices. The patients fasted for at least 6 hours before the PET/CT examination. The patients' blood glucose level was checked and controlled at $<6.8 \mathrm{mmol} / \mathrm{L}$ before $18 \mathrm{~F}$-fluorodeoxyglucose injection (3.7 to $4.5 \mathrm{MBq} / \mathrm{kg}$ body weight), which was performed after micturition and resting for 60 minutes. Images were obtained from the base of the skull to the mid-thigh level. The SUVmax of each primary tumor and suspicious lymph node station was determined 
by drawing a region of interest around it. The qualitative and quantitative results of the PET/CT scans, such as the SUVmax of the primary tumor and those of the lymph nodes, were recorded by two nuclear medicine physicians who were unaware of the pathological findings. In subsolid tumors, the solid diameter of the nodule was recorded for tumor size. Pathological staging followed the eighth edition of the TNM staging manual. The pathological findings served as the gold standard for comparison with the PET/ CT findings.

\section{Blood parameters}

The serum levels of cancer antigen 199 (CA199), carcinoembryonic antigen (CEA), cancer antigen 125 (CA125) and squamous cell carcinoma antigen (SCCA) were measured, with reference thresholds of $25.0 \mathrm{ng} / \mathrm{mL}$, $5.0 \mathrm{ng} / \mathrm{mL}, 35.0 \mathrm{U} / \mathrm{mL}$ and $1.5 \mathrm{ng} / \mathrm{mL}$, respectively. The CA199, SCCA, and CA125 levels were then excluded because few of these tumor markers exceeded their respective thresholds in the patients sampled, which left the CEA level as our predictive factor. A routine blood examination with automated differential counts was performed in every patient during the week before surgery. The numbers of leukocytes, neutrophils, and lymphocytes were also assessed. Patients with a total leukocyte count greater than $10,000 / \mu \mathrm{L}$ or less than $4,000 / \mu \mathrm{L}$ were removed from our study. Finally, the ratio of neutrophils to lymphocytes (NLR) was calculated.

\section{Statistical analysis}

IBM SPSS for Windows (v24.0) and R 3.5.0 were used for statistical analysis. The PET/CT results were compared with the pathological findings. Univariate and multivariable logistic regression was performed, and an odds ratio (OR) with a $95 \%$ confidence interval (CI) was used to estimate correlation strength. Predictors $(\mathrm{P}<0.1)$ in univariable analysis were entered a multivariable regression analysis. Tumor histologic type is huge confounders that were also adjusted in the multivariable analysis. A nomogram capable of predicting the risk of lymph node metastasis was then developed based on the final multivariable analysis and excellent log-likelihood ratio analysis. The performance of the nomogram was assessed by discrimination and calibration. The area determined the model's discriminative ability under the receiver operating characteristic curve, which ranged from
0.5 (no discrimination) to 1 (perfect discrimination) (17). The Hosmer-Lemeshow test evaluated the calibration capability of predictive models. The nomogram was subjected to 1,000 iterations of bootstrap resampling for internal validation and independent validation to assess its predictive accuracy (18). In addition, decision curve analysis was used to determine the clinical usefulness of the nomogram by quantifying the net benefits at different threshold probabilities in the primary dataset.

\section{Results}

The characteristics of the enrolled patients are shown in Table 1. Lymph node metastasis was pathologically confirmed after surgery in 113 cases $(35.5 \%)$ in the primary cohort (95 showed N1 metastasis and 69 showed N2 metastasis) and 44 cases (28.2\%) in the validation cohort (29 showed N1 metastasis and 27 showed N2 metastasis). Four hundred and twenty (88.4\%) patients underwent minimally invasive approach. The median age of the reviewed patients was 63 years (range, 34 to 81 years old) in primary cohort, and 68 years (range, 33 to 81 years old) in the validation cohort; 155 were male $(48.7 \%)$ and 163 were female $(51.3 \%)$ in primary cohort, 86 were male $(55.1 \%)$ and 70 were female $(44.9 \%)$ in validation cohort. Tumor location was confirmed by CT scan and bronchoscopy; centraltype pulmonary carcinomas have a high rate of lymph node involvement with malignancy. The results of the univariate analysis indicate that the sex, age, grade, and CEA were associated with nodal metastasis in the primary cohort, the tumor location, tumor size, lymph node short-axis size, tumor SUVmax, and lymph node SUVmax were associated with nodal metastasis in both cohorts.

According to the results of our final analysis in primary cohort (Table 2), age (OR, 0.953; 95\% CI, 0.921 to 0.988 ; $\mathrm{P}=0.008$ ), tumor location (OR, 7.018; 95\% CI, 2.687 to 18.330; $\mathrm{P}<0.001)$, histology $(\mathrm{P}=0.007)$, tumor $\mathrm{SUVmax}$ (OR, 1.126; 95\% CI, 1.040 to $1.220 ; \mathrm{P}=0.004$ ), lymph node SUVmax (OR, 1.436; 95\% CI, 1.201 to 1.718 ; $\mathrm{P}<0.001$ ) are independent hazardous factors for lymph node metastasis in patients with NSCLC. Based on the log-likelihood ratio achieved via step-down selection, the CEA value also was entered into the final regression analysis. Age, tumor location, histology, tumor SUVmax, lymph node SUVmax, and CEA value were chosen to construct a nomogram to predict lymph node metastasis in patients with NSCLC (Figure 1). The area under the receiver operating characteristic curve was 0.858 (95\% CI, 0.815 to 0.901$)$ in 
Table 1 Patient characteristics and univariate analysis of the risk of lymph node metastasis

\begin{tabular}{|c|c|c|c|c|c|c|}
\hline Variables & \multicolumn{3}{|c|}{ Primary cohort $(n=318)$} & \multicolumn{3}{|c|}{ Validation cohort $(n=156)$} \\
\hline Sex & & & 0.011 & & & 0.327 \\
\hline Male & $89(43.4)$ & $66(58.4)$ & & $59(52.7)$ & $27(61.4)$ & \\
\hline Mean (SD) & $63.9(9.6)$ & $60.5(9.4)$ & & $63.2(8.6)$ & $62.7(9.7)$ & \\
\hline Tumor location & & & $<0.001$ & & & 0.021 \\
\hline Peripheral & $189(92.2)$ & $73(64.6)$ & & $97(86.6)$ & $31(70.5)$ & \\
\hline Yes & $68(33.2)$ & $49(43.4)$ & & $50(44.6)$ & $24(54.5)$ & \\
\hline Tumor size, $\mathrm{cm}$ & & & $<0.001$ & & & 0.004 \\
\hline Mean (SD) & $2.2(1.1)$ & $2.9(0.8)$ & & $2.5(1.3)$ & $3.8(3.4)$ & \\
\hline Histology & & & 0.637 & & & 0.049 \\
\hline Adenocarcinoma & $165(80.5)$ & $87(77.0)$ & & $93(83.0)$ & $31(70.5)$ & \\
\hline Squamous cell carcinoma & $25(12.2)$ & $18(15.9)$ & & $16(14.3)$ & $9(20.5)$ & \\
\hline Other & $15(7.3)$ & $8(7.1)$ & & $3(2.7)$ & $4(9.1)$ & \\
\hline Mean (SD) & $0.5(0.4)$ & $0.8(0.5)$ & & $0.8(0.4)$ & $1.0(0.4)$ & \\
\hline Tumor SUVmax value & & & $<0.001$ & & & $<0.001$ \\
\hline Mean (SD) & $4.7(4.7)$ & $9.2(4.4)$ & & $5.6(4.3)$ & $8.7(4.5)$ & \\
\hline Lymph node SUVmax value & & & $<0.001$ & & & $<0.001$ \\
\hline Mean (SD) & $1.7(2.0)$ & $4.0(3.1)$ & & $2.1(2.0)$ & $3.8(3.2)$ & \\
\hline CEA value, $\mathrm{ng} / \mathrm{mL}$ & & & 0.003 & & & 0.030 \\
\hline Mean (SD) & $5.0(7.0)$ & $8.1(9.5)$ & & $5.5(9.3)$ & $12.4(23.7)$ & \\
\hline Neutrophil/lymphocyte value & & & 0.200 & & & 0.487 \\
\hline Mean (SD) & $2.2(1.1)$ & $2.4(1.2)$ & & $3.0(3.2)$ & $2.7(2.1)$ & \\
\hline Ground glass opacity & & & 0.997 & & & 0.998 \\
\hline No & $149(72.7)$ & $113(100.0)$ & & $97(86.6)$ & $44(100.0)$ & \\
\hline Yes & $56(27.3)$ & $0(0.0)$ & & $15(13.4)$ & $0(0.0)$ & \\
\hline
\end{tabular}

$P$ value is derived from the univariable analyses between each variable and node status. 
Table 2 The variables identified by logistic multivariable regression analysis

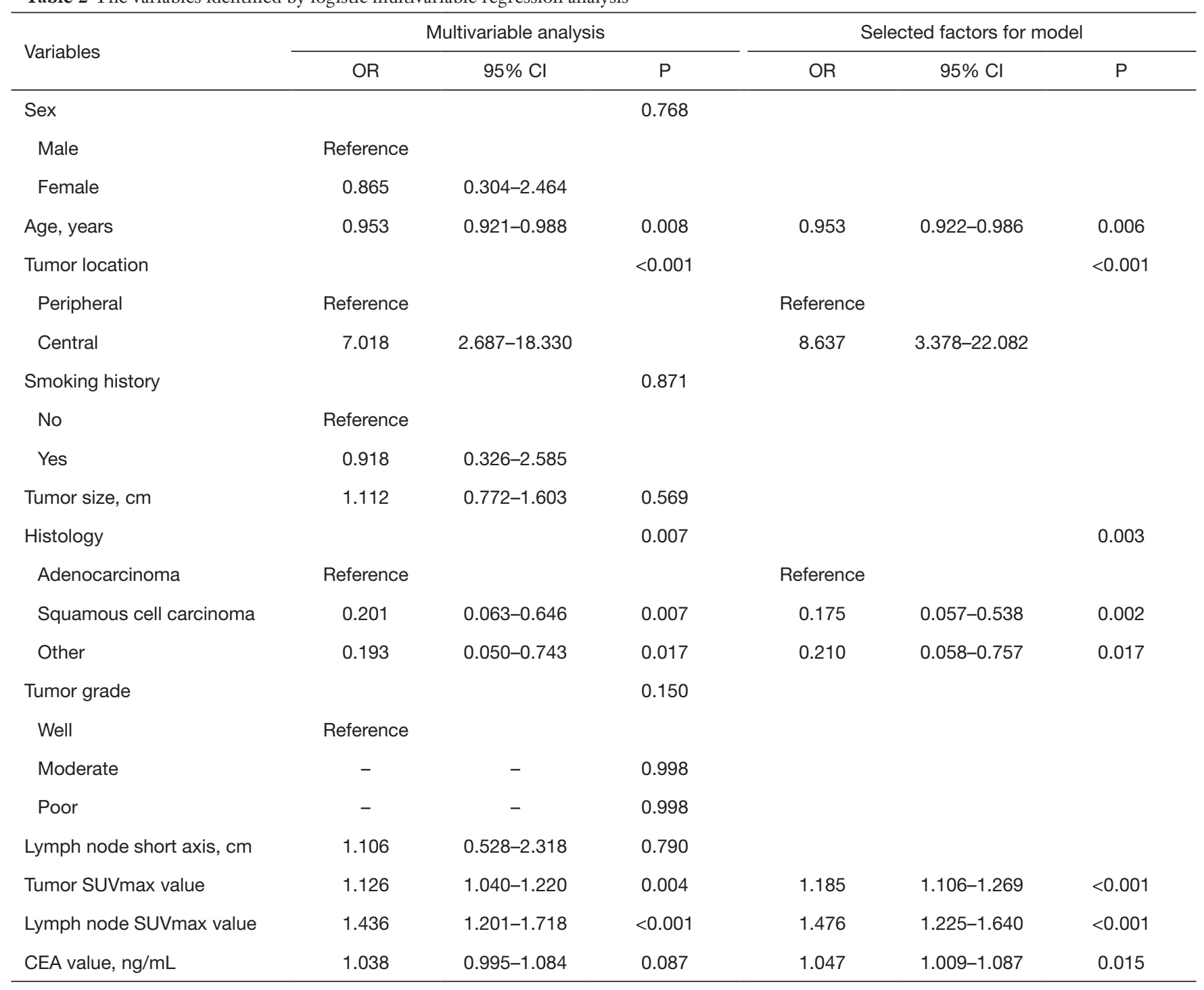

OR, odds ratio; $\mathrm{Cl}$, confidence interval; CEA, carcinoembryonic antigen.

the primary cohort and was 0.749 (95\% CI, 0.660 to 0.838$)$ in the validation cohort, indicating robust discrimination. The Hosmer-Lemeshow test showed good calibration capability $(\mathrm{P}=0.517$ in the primary cohort, and $\mathrm{P}=0.642$ in the validation cohort). The nomogram calibration curves for the probability of lymph node metastasis showed excellent concordance between the predicted and actual results (Figure 2). The decision curve showed that if the threshold probability of a patient is between $5 \%$ and $95 \%$, using the nomogram to predict lymph node metastasis adds more benefit than either the treat-all or treat-none scheme (Figure 3).

\section{Discussion}

In this retrospective study, we constructed and confirmed a nomogram to combine PET/CT results with clinical characteristics to predict the risk of nodal metastasis in patients with T1-2 NSCLC. We identified 6 factors that were predictive of lymph node metastasis: age, tumor location, histology, tumor SUVmax, lymph node SUVmax, and CEA value. Our nomogram proved that younger NSCLC patients with central lung adenocarcinoma cancer and higher tumor SUVmax, lymph node SUVmax and CEA values had a greater risk of nodal involvement with NSCLC.

Several models have been developed to predict nodal 


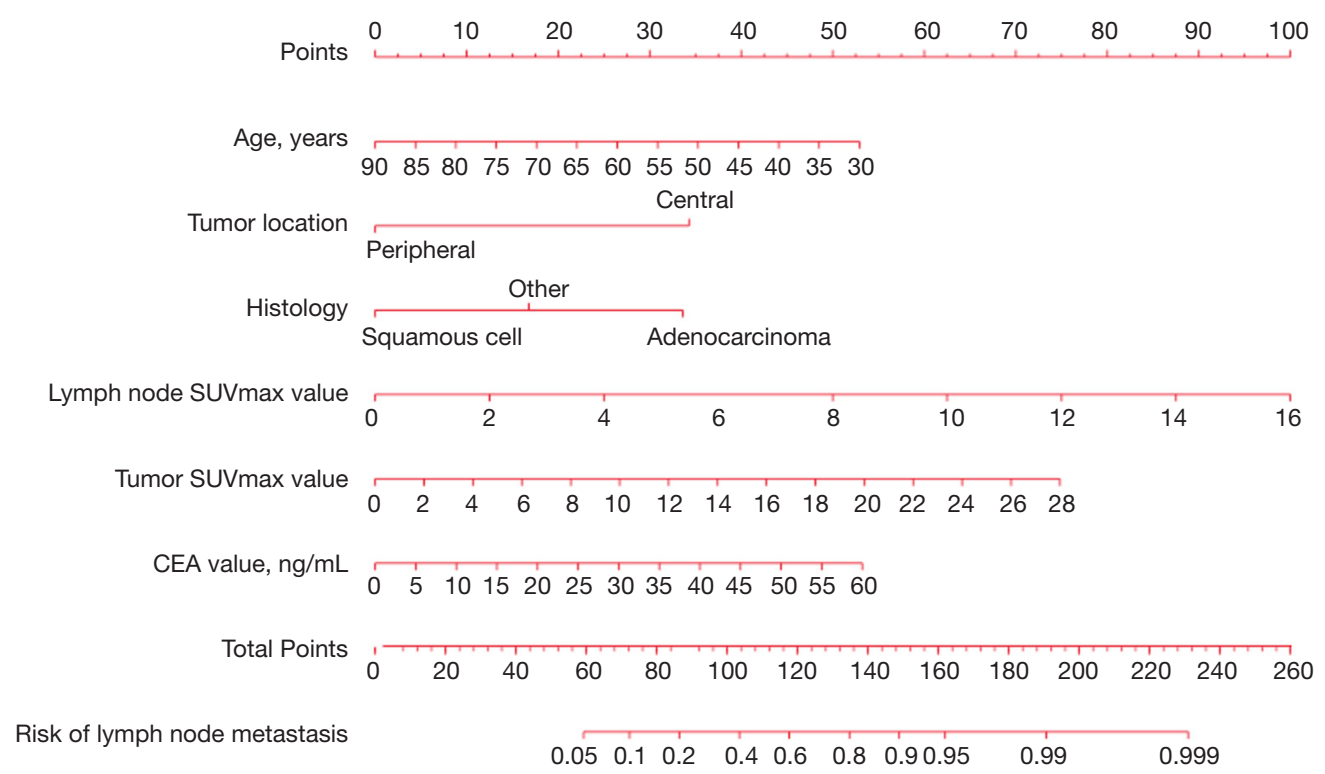

Figure 1 Nomogram for predicting the risk of lymph nodes metastasis in non-small-cell lung cancer. The value of each variable was given a score on the point scale axis. A total score could be easily calculated by adding every single score and, by projecting the total score to the lower total point scale, we were able to estimate the probability of lymph nodes metastasis.
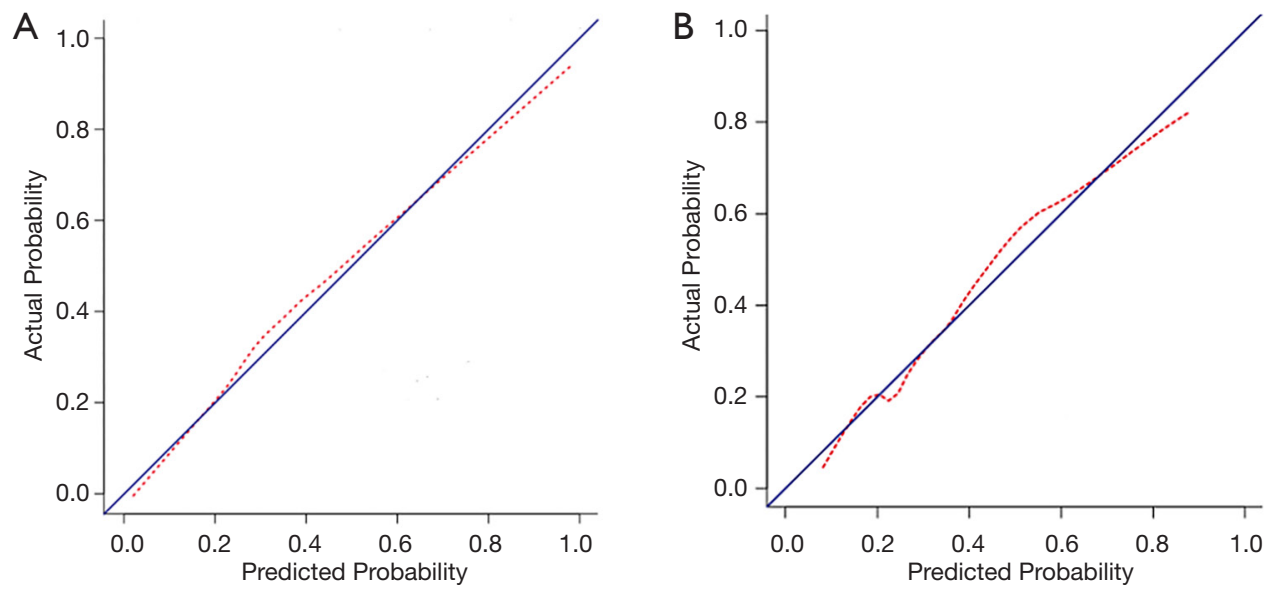

Figure 2 The calibration curves for the nomogram. (A) Primary cohort; (B) validation cohort. The x-axis represents the nomogram predicted probability, and the y-axis represents the actual probability of lymph nodes metastasis. A perfect prediction would correspond to the diagonal blue solid line. The red dashed line represents the performance of the nomogram, of which a closer fit to the diagonal line represents a better prediction.

involvement with malignancy in NSCLC $(8,9,15,16,19)$. However, only two prediction models have been established to estimate the pretest probability of N2 lymph nodes metastasis, and one model has been built to predict the lymph node involvement in patients with NSCLC based on PET/CT $(9,15,16)$. We extracted key factors from the clinical data and preoperative common examination results to develop an effective procedure for the assessment of lymph node metastasis. In contrast with earlier models, age and CEA values were incorporated into our nomogram due to their significance as indicated by multivariable analysis.

Recently, a retrospective study of 2,237 patients with 


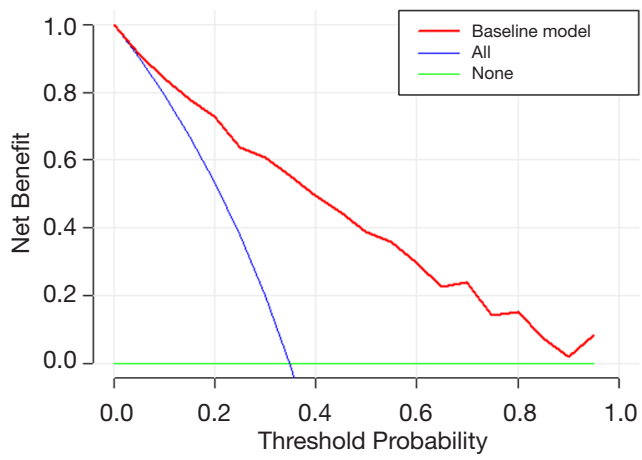

Figure 3 Decision curve analysis for the nomogram. The y-axis measures the net benefit. The red line represents the nomogram. The blue line represents the assumption that all patients have lymph nodes metastasis. The green line represents the assumption that no patients have lymph nodes metastasis. The net benefit was calculated by subtracting the proportion of all patients who are false positive from the proportion, which is a true positive.

NSCLC revealed that younger patients were more likely to harbor genotype (EGFR, ALK, HER2, ROS1) alteration; unexpectedly, their study showed that patients older than 70 years of age and younger than 40 years of age had lower survival rates than other age groups (20). Because younger patients often have a lower rate of co-morbidity and functional impairment, their higher probability of harboring targetable genotype alteration may relate to younger patients' failure to show a higher survival rate than the older patients. Genotype alteration has been reported to relate to lymph node metastasis $(21,22)$. In some ways, these findings are consistent with those of our study: our results for age as an independent predictive factor in lymph node assessment indicate that younger patients had a higher OR for nodal metastasis than older patients. Younger NSCLC patients have a higher rate of targetable genotype alteration or more aggressive disease biology.

The role of CEA as a tumor maker has already been acknowledged, and the use of this factor to predict prognosis in patients with NSCLC has been widely studied. High CEA levels both before and after surgery are associated with a shorter duration of disease-free survival $(23,24)$. Nosotti and colleagues used micrometastatic analysis to prove that patients with lymph nodes negative for CEA mRNA showed a lower rate of lung cancer recurrence (25). Given these results, we were unsurprised to find that the CEA level was a predictive factor in our model. Our study also indicated that the CEA level should be considered before surgery.
According to numerous studies, tumor location relates to nodal involvement with malignancy; specifically, compared with peripheral tumors, central lung cancer has a significantly higher prevalence of lymph node metastasis (26-28). Tumor histologic type also has been identified to be associated with lymph node metastasis in patients with NSCLC. We integrated the histology into the model and found adenocarcinoma histology as a risk factor for lymph node involvement, which agrees with most of the published literature $(8,9,16)$. In addition, PET/CT has been widely reported to assess nodal involvement more accurately with malignancy than CT scans, usually based on the criterion of lymph node SUVmax. Several retrospective studies have shown that patients with mediastinal lymph node metastasis have a significantly higher median SUVmax in the primary tumor than patients without lymph node metastasis (29-31), suggesting an association between tumor SUVmax and lymph node metastasis. Lymph node SUVmax and primarytumor SUVmax were both incorporated into our model, and the former was found to make a greater contribution to the risk of nodal metastasis.

Notably, lymph node short-axis dimensions, as revealed by CT imaging, are an important parameter in the assessment of nodal malignancy, although this parameter is unreliable due to its relative lack of accuracy in the assessment of lymph nodes with small lesions (26). However, our multivariable analysis revealed no association between lymph node shortaxis size and lymph node metastasis, consistent with the results of Mattes (16). Univariate analysis indicated that the NLR and ground-glass opacity had no significant effect on the risk of lymph node metastasis, although these factors have previously been reported to be associated with regional nodal involvement with malignancy in patients with NSCLC $(10,32)$. We recommend further research to corroborate the connection between these factors and nodal metastasis in patients with NSCLC.

Several limitations of this study should be addressed. First, the retrospective nature and limited samples of the study may therefore introduce the possibility of some inevitable selection bias. We attempted to balance the apparent biases by using multivariate analysis, but there are still some potential biases between the groups that were not known. Second, PET/CT scanning is not a routine examination procedure in our department, and we reviewed only the records of patients who had undergone PET/CT scanning and excluded those with few clinical manifestations who had not. Third, although internal and independent validation can minimize these adverse effects and improve 
the calibration of the predictive model, the generalisability of the nomogram must still be tested using data from other countries to accommodate variation between ethnic groups. The use of more non-imaging data and patient records with a wider geographical range is expected to optimize the nomogram for future use.

In conclusion, we have established and validated a novel effective nomogram capable of predicting the risk of lymph node metastasis for individual patients with T1-2 NSCLC. This model offers a more effective and noninvasive preoperative means of assessing patients' risk of lymph node metastasis and may thus help clinicians to make individual treatment recommendations.

\section{Acknowledgments}

Funding: This work was supported by the Key Project of Zhejiang Province Science and Technology Plan, China (2014C03032), and the National Key R\&D Program of China (2017YFC0113500).

\section{Footnote}

Reporting Checklist: The authors have completed the TRIPOD reporting checklist. Available at http://dx.doi. org/10.21037/tlcr-20-1026

Data Sharing Statement: Available at http://dx.doi. org/10.21037/tlcr-20-1026

Conflicts of Interest: All authors have completed the ICMJE uniform disclosure form (available at http://dx.doi. org/10.21037/tlcr-20-1026). The authors have no conflicts of interest to declare.

Ethical Statement: The authors are accountable for all aspects of the work in ensuring that questions related to the accuracy or integrity of any part of the work are appropriately investigated and resolved. The study was performed following the principles outlined in the Declaration of Helsinki (as revised in 2013). The study was approved by the university's ethical committee and institutional review board. (No. 2020759) and written informed consent was obtained from all patients.

Open Access Statement: This is an Open Access article distributed in accordance with the Creative Commons Attribution-NonCommercial-NoDerivs 4.0 International
License (CC BY-NC-ND 4.0), which permits the noncommercial replication and distribution of the article with the strict proviso that no changes or edits are made and the original work is properly cited (including links to both the formal publication through the relevant DOI and the license). See: https://creativecommons.org/licenses/by-nc-nd/4.0/.

\section{References}

1. Smeltzer MP, Faris NR, Ray MA, et al. Association of pathologic nodal staging quality with survival among patients with non-small cell lung cancer after resection with curative intent. JAMA Oncol 2018;4:80-7.

2. Asamura H, Chansky K, Crowley J, et al. The International Association for the Study of Lung Cancer lung cancer staging project: proposals for the revision of the $\mathrm{N}$ descriptors in the forthcoming 8 th edition of the TNM classification for lung cancer. J Thorac Oncol 2015;10:1675-84.

3. Onaitis MW, Furnary AP, Kosinski AS, et al. Prediction of long-term survival after lung cancer surgery for elderly patients in the Society of Thoracic Surgeons General Thoracic Surgery Database. Ann Thorac Surg 2018;105:309-16.

4. Chang JY, Senan S, Paul MA, et al. Stereotactic ablative radiotherapy versus lobectomy for operable stage I nonsmall- cell lung cancer: a pooled analysis of two randomized trials. Lancet Oncol 2015;16:630-7.

5. Bang A, Bezjak A. Stereotactic body radiotherapy for centrally located stage I non-small cell lung cancer. Transl Lung Cancer Res 2019;8:58-69.

6. Truong MT, Viswanathan C, Erasmus JJ. Positron emission tomography/computed tomography in lung cancer staging, prognosis, and assessment of therapeutic response. J Thorac Imaging 2011;26:132-46.

7. Lv YL, Yuan DM, Wang K, et al. Diagnostic performance of integrated positron emission tomography/computed tomography for mediastinal lymph node staging in nonsmall cell lung cancer: a bivariate systematic review and meta-analysis. J Thorac Oncol 2011;6:1350-8.

8. Zhang Y, Sun Y, Xiang J, et al. A prediction model for $\mathrm{N} 2$ disease in T1 non-small cell lung cancer. J Thorac Cardiovasc Surg 2012;144:1360-4.

9. Farjah F, Lou F, Sima C, et al. A prediction model for pathologic $\mathrm{N} 2$ disease in lungcancer patients with a negative mediastinum by positron emission tomography. J Thorac Oncol 2013;8:1170-80.

10. Roengvoraphoj O, Käsmann L, Eze C, et al. Maximum 
standardized uptake value of primary tumor (SUVmax_PT) and horizontal range between two most distant PET-positive lymph nodes predict patient outcome in inoperable stage III NSCLC patients after chemoradiotherapy. Transl Lung Cancer Res 2020;9:541-8.

11. Hellwig D, Graeter TP, Ukena D, et al. 18F-FDG PET for mediastinal staging of lung cancer: which SUV threshold makes sense? J Nucl Med 2007;48:1761-6.

12. YangW, Fu Z, Yu J, et al. Value of PET/CT versus enhanced CT for locoregional lymph nodes in non-small cell lung cancer. Lung Cancer 2008;61:35-43.

13. Jin C, Cao J, Cai Y, et al. A nomogram for predicting the risk of invasive pulmonary adenocarcinoma for patients with solitary peripheral subsolid nodules. J Thorac Cardiovasc Surg 2017;153:462-9.e1.

14. Mao Q, Xia W, Dong G, et al. A nomogram to predict the survival of stage IIIA-N2 non-small cell lung cancer after surgery. J Thorac Cardiovasc Surg 2018;155:1784-92.e3.

15. Jiang L, Jiang S, Lin Y, et al. Nomogram to predict occult $\mathrm{N} 2$ lymph nodes metastases in patients with squamous non-small cell lung cancer. Medicine (Baltimore) 2015;94:e2054.

16. Mattes MD, Weber WA, Foster A, et al. A predictive model for lymph node involvement with malignancy on PET/CT in non-small-cell lung cancer. J Thorac Oncol 2015;10:1207-12.

17. Bandos AI, Rockette HE, Song T, et al. Area under the free-response ROCcurve (FROC) and a related summary index. Biometrics 2009;65:247-56.

18. Iasonos A, Schrag D, Raj GV, et al. How to build and interpret a nomogram for cancer prognosis. J Clin Oncol 2008;26:1364-70.

19. Gu Y, She Y, Dai C, et al. A texture analysis-based prediction model for lymph node metastasis in stage IA lung adenocarcinoma. Ann Thorac Surg 2018;106:214-20.

20. Sacher AG, Dahlberg SE, Heng J, et al. Association between younger age and targetable genomic alterations and prognosis in non-small-cell lung cancer. JAMA Oncol 2016;2:313-20.

21. Han CB, Ma JT, Li F, et al. EGFR and KRAS mutations and altered c-Met gene copy numbers in primary nonsmall cell lung cancer and associated stage N2 lymph node-metastasis. Cancer Lett 2012;314:63-72.

22. Schmid K, Oehl N, Wrba F, et al. EGFR/KRAS/ BRAF mutations in primary lung adenocarcinomas and corresponding locoregional lymph node metastases. Clin Cancer Res 2009;15:4554-60.

23. Grunnet M, Sorensen JB. Carcinoembryonic antigen (CEA) as tumor marker in lung cancer. Lung Cancer
2012;76:138-43.

24. Matsuguma H, Nakahara R, Igarashi S, et al. Pathologic stage I non-small cell lung cancer with high levels of preoperative serum carcinoembryonic antigen: linicopathologic characteristics and prognosis. J Thorac Cardiovasc Surg 2008;135:44-9.

25. Nosotti M, Falleni M, Palleschi A, et al. Quantitative realtime polymerase chain reaction detection of lymph node lung cancer micrometastasis using carcinoembryonic antigen marker. Chest 2005;128:1539-44.

26. Decaluwé H, Stanzi A, Dooms C, et al. Central tumor location should be considered when comparing N1 upstaging between thoracoscopic and open surgery for clinical stage I non-small-cell lung cancer. Eur J Cardiothorac Surg 2016;50:110-7.

27. Al-Sarraf N, Aziz R, Gately K, et al. Pattern and predictors of occult mediastinal lymph node involvement in non-small cell lung cancer patients with negative mediastinal uptake on positron emission tomography. Eur J Cardiothorac Surg 2008;33:104-9.

28. Lee PC, Port JL, Korst RJ, et al. Risk factors for occult mediastinal metastases in clinical stage I non-small cell lung cancer. Ann Thorac Surg 2007;84:177-81.

29. Peng Z, Liu Q, Li M, et al. Comparison of (11) C-choline PET/CT and enhanced CT in the evaluation of patients with pulmonary abnormalities and locoregional lymph node involvement in lung cancer. Clin Lung Cancer 2012;13:312-20.

30. Mattes MD, Moshchinsky AB, Ahsanuddin S, et al. Ratio of lymph node to primary tumor SUV on PET/CT accurately predicts nodal malignancy in non-small-cell lung cancer. Clin Lung Cancer 2015;16:e253-8.

31. Nambu A, Kato S, Sato Y, et al. Relationship between maximum standardized uptake value (SUVmax) of lung cancer and lymph node metastasis on FDG-PET. Ann Nucl Med 2009;23:269-75.

32. Huang C, Yue J, Li Z, et al. Usefulness of the neutrophilto-lymphocyte ratio in predicting lymph node metastasis in patients with non-small cell lung cancer. Tumour Biol 2015;36:7581-9.

Cite this article as: $\mathrm{Lv} \mathrm{X}, \mathrm{Wu} \mathrm{Z}$, Cao J, Hu Y, Liu K, Dai X, Yuan X, Wang Y, Zhao K, Lv W, Hu J. A nomogram for predicting the risk of lymph node metastasis in T1-2 non-smallcell lung cancer based on PET/CT and clinical characteristics. Transl Lung Cancer Res 2021;10(1):430-438. doi: 10.21037/tlcr20-1026 